\title{
Caracterización y calidad de los compost producidos y comercializados en Rupa Rupa-Huánuco
}

\section{(Characterization and quality of compost produced and marketed in Rupa Rupa-Huánuco)}

\author{
Marlon Ricardo, Bailón-Rojas ${ }^{1}$, Nelino, Florida-Rofner ${ }^{2}$
}

\begin{abstract}
Resumen
El cacao y el café son cultivos importantes en la zona que se manejan bajo normas de producción orgánica, y el uso de abonos orgánicos es alto, en especial del compost. Por ello, se evaluaron los indicadores fisicoquímicos y se determinó la calidad de los compost producidos con diferentes insumos y comercializados en el distrito Rupa Rupa, región Huánuco-Perú. Es una investigación no experimental correlacional con ajuste estadístico completamente aleatorizado; los tratamientos lo constituyen los siguientes compost identificados: compost Alborada (CAL), compost M\&F Orgánicos (CMF), compost de residuos municipal (CML), compost de la cooperativa Divisoria (CCD) y compost comercial formulado (CCF). Se evaluaron indicadores físicos (humedad) y químicos (cenizas, $\mathrm{pH}, \mathrm{MO}, \mathrm{N}, \mathrm{P}_{2} \mathrm{O}_{5}, \mathrm{~K}, \mathrm{Ca}, \mathrm{Mg}, \mathrm{Na}, \mathrm{Cu}$, Fe, Mn y Zn) y se determinó la calidad según la norma técnica colombiana (NTC 5167), la Norma Oficial Chilena (NOCh 2880) y los estándares de calidad de la Organización Mundial de la Salud (OMS). Los resultados no muestran diferencias en los indicadores fisicoquímicos, excepto los niveles de N y, según la NTC y la OMS, corresponden a compost de buena calidad, mientras la NOCh los clasifica como compost de calidad media. En conclusión, los compost evaluados son de calidad media y la norma que mejor determina la calidad es la NOCh, que puede ser aplicada en países que no disponen de una norma específica que determine la calidad del compost.
\end{abstract}

\section{Palabras clave}

Compost formulado, compost estable, estándares de calidad, indicadores de calidad, normas técnicas, residuo municipal.

\begin{abstract}
Cocoa and coffee are important crops in the area and are managed under organic production standards, and the use of organic fertilizers is high, especially compost. For this reason, physical-chemical indicators were evaluated, and the quality of the compost produced with different inputs and marketed in the Rupa Rupa district, Huánuco-Peru region, was determined. It is a non-experimental correlational investigation with statistical adjustment of completely randomized, the treatments are the identified compost: Alborada compost (CAL), M\&F Organic compost (CMF), municipal waste compost (CML), compost from the Divisoria Cooperative (CCD) and formulated commercial compost (CCF). Physical (humidity) and chemical indicators (ashes, pH, MO, N, P205, K, Ca, Mg, Na, Cu, Fe, Mn and Zn) were evaluated and the quality was determined according to the Colombian technical standard (NTC 5167), the Chilean official standard (NOCh 2880) and the quality standards of the World Health Organization (OMS). The Results show no differences in the physical and chemical indicators, except for the N levels and according to the NTC and the OMS correspond to good quality compost and the NOCh classifies them as medium quality compost. In conclusion, the compost evaluated are of medium quality and the standard that best determines the quality is the NOCh, which can be applied in countries that do not have a specific standard that determines the quality of the compost.
\end{abstract}

\section{Keywords}

Formulated compost, stable compost, quality standards, technical norms, municipal waste

1 Universidad Nacional Agraria de la Selva, Tingo María Huánuco-Perú. ORCID: https://orcid.org/0000-0001-9907-5210, email: bailon84@hotmail.com

2 Universidad Nacional Agraria de la Selva, Tingo María Huánuco-Perú. ORCID: https://orcid.org/0000-0002-8751-4367, email: nelinof@hotmail.com 


\section{Introducción}

Los cultivos de importancia económica en el distrito Rupa Rupa, provincia de Leoncio Prado, son el café y el cacao. Al respecto, el Ministerio de Agricultura y Riego (MINAGRI, 2019a) informa que Perú es el segundo exportador mundial de café orgánico, que es el primer producto agrícola peruano de exportación, y el séptimo país exportador de café a nivel mundial y posee 425416 hectáreas, que representan $6 \%$ del área agrícola nacional; mientras que Huánuco es el séptimo productor a nivel nacional. Además, el segundo cultivo más importante en la provincia es el de cacao que a nivel nacional, en 2018, alcanzó una extensión de 199 mil hectáreas. Huánuco ocupa el quinto lugar en extensión de este cultivo (MINAGRI, enero 2019b), del cual el 75 \% es calificado cacao fino o de aroma, por lo que, en 2015, ocupó el tercer lugar como país productor de cacao fino o de aroma en el mundo (MINAGRI, 2016), y, en 2018, se posicionó como segundo productor de cacao orgánico a nivel mundial (MINAGRI, 2019c).

El cacao y el café son los cultivos más importantes en el área de influencia de esta investigación y se manejan bajo las normas de una producción orgánica, impulsada por diferentes instituciones públicas, privadas y por asociaciones de productores; además, el uso de abonos orgánicos en el proceso de producción es alto, en especial del compost. La calidad de estos productos orgánicos es relevante, por ello, la evaluación de sus principales indicadores físicoquímicos como humedad, olor, color $\mathrm{pH}, \mathrm{MO}, \mathrm{N}, \mathrm{P}$ y bases como $\mathrm{Ca}$, $\mathrm{K}$ y Mg permiten medir la calidad (Hernández, 2020; Rivas \& Silva, 2020; Florida \& Reategui, 2019; Rafael, 2015; García et al., 2014; Organización de las Naciones Unidas para la Alimentación y la Agricultura [FAO, por sus siglas en inglés] 2013; Mendoza, 2012) y la madurez de un material compostado (Muscolo et al., 2018). El conocimiento de la calidad, expresado en los indicadores fisicoquímicos, es fundamental, independientemente de la madurez, para mantener la sostenibilidad económica y ambiental en todo proceso productivo.

El compostaje es una técnica que permite la biodegradación controlada de residuos y subproductos orgánicos (Rivas \& Silva, 2020; López et al., 2017), transformándolos en materiales biológicamente estables cuyo producto final es el compost, fertilizante de liberación lenta con efectos residuales positivos (Peralta et al., 2019; Sharma et al., 2017) usado como sustrato (Bárbaro et al., 2019) y con capacidad de mejorar las condiciones edáficas y la producción del cultivo (Cabrera \& Rossi, 2016). Como materia orgánica estabilizada, se puede usar para recuperar suelos degradados, restaurar la fertilidad reduciendo el uso de insumos químicos tradicionales (Rivas \& Silva, 2020; Alvarez et al., 2019) y disminuir la disposición de residuos en vertederos (Florida \& Reategui, 2019; Muscolo et al., 2018).

El compost sin un control de calidad de la autoridad competente puede generar efectos perjudiciales a los agroecosistemas y a la economía de los productores, por la baja capacidad fertilizante o por la presencia de sustancias fitotóxicas en elevadas concentraciones. Por tanto, su utilización es altamente riesgosa en los cultivos (García et al., 2014); frente a ello, Ramos \& Terry (2014) sugieren cumplir con determinadas normas de calidad fijada por cada país, rangos que el material compostado debe cumplir (Rafael, 2015). Sin embargo, Perú carece de una norma técnica específica para definir la calidad del compost y recurre a las principales normas de países vecinos como la norma técnica colombiana NTC 5167 (Instituto Colombiano de Normas Técnicas y Certificación, 2011) y la Norma Oficial Chilena NOCh 2880 (Instituto Nacional de Normalización, 2004) y, complementariamente, a los estándares de la OMS y la FAO. En este contexto, la investigación planteó como objetivo evaluar los indicadores fisicoquímicos y determi- 
nar la calidad, con base en la NOCh 2880, NTC 5167 y los estándares de la OMS, de los compost que se producen y comercializan en el distrito Rupa Rupa, región Huánuco, Perú.

\section{Metodología}

\section{Área de estudio}

El lugar donde se desarrolló la investigación está en la jurisdicción de la ciudad de Tingo María, que pertenece políticamente al distrito de Rupa Rupa, provincia de Leoncio Prado, región Huánuco-Perú. Las condiciones climáticas generales que presenta, según el Servicio Nacional de Meteorología e Hidrología del Perú (2020), son las siguientes: temperatura media anual de 24.5 C, humedad relativa media anual de 84 \% y precipitación media anual de 3400 mm. Según Pulgar (2014), el área pertenece a la ecorregión Rupa Rupa o selva alta.

\section{Sobre los tipos de abonos}

La investigación identificó las empresas, asociaciones y dueños de fincas que producen abonos orgánicos en la ciudad de Tingo María, Castillo Grande y Naranjillo, así como aquellos que ofrecen comercialmente (compost formulados). Se hizo una descripción de los insumos básicos de su preparación y se analizó por cuadruplicado (4 muestras) de cada uno de los abonos identificados y seleccionados, entre ellos:

a. Abono orgánico tipo compost: producido por la Cooperativa Agraria Cafetalera Divisoria (CCD). La empresa no aceptó develar los insumos que utiliza en su producción, sin embargo, el producto presenta su ficha técnica (Tabla 1) que describe su composición.

Tabla 1. Ficha técnica, compost de la Cooperativa Cafetalera Divisoria (CCD)

\begin{tabular}{|c|c|c|}
\hline Indicador & Unidad & Contenido medio \\
\hline Humedad & $\%$ & 18 \\
\hline MO & $\%$ & 40 \\
\hline N & $\%$ & $1.2 \pm 0.5$ \\
\hline P205 & $\%$ & $1.5 \pm 0.5$ \\
\hline $\mathrm{Ca}$ & $\%$ & $9.5 \pm 0.5$ \\
\hline $\mathrm{MgO}$ & $\%$ & $5.5 \pm 0.5$ \\
\hline $\mathrm{K} 20$ & $\%$ & $1.5 \pm 0.5$ \\
\hline $\mathrm{Na}$ & $\%$ & 0.5 \\
\hline $\mathrm{Cu}$ & $\mathrm{ppm}$ & 42 \\
\hline $\mathrm{Fe}$ & $\mathrm{ppm}$ & 3029 \\
\hline $\mathrm{Zn}$ & $\mathrm{ppm}$ & 780 \\
\hline $\mathrm{Mn}$ & $\mathrm{ppm}$ & 1647 \\
\hline
\end{tabular}


b. Compost producidos por la empresa M\&F Orgánicos (CMF): la Tabla 2 describe su composición media; según el productor, el compost que produce es 100 \% a base de estiércol de vacuno y mediado por microorganismos eficientes.

Tabla 2. Ficha técnica del compost producido por la empresa M\&F Orgánicos (CMF)

\begin{tabular}{|c|c|c|}
\hline Indicador & Unidad & Contenido medio \\
\hline Humedad & $\%$ & 20 \\
\hline Cenizas & $\%$ & -- \\
\hline $\mathrm{pH}$ & $\% .82$ \\
\hline $\mathrm{MO}$ & $\%$ & 39 \\
\hline $\mathrm{N}$ & $\%$ & 1.7 \\
\hline $\mathrm{P} 205$ & $\%$ & 1 \\
\hline $\mathrm{Ca}$ & $\%$ & 1.9 \\
\hline $\mathrm{MgO}$ & $\%$ & 1.4 \\
\hline $\mathrm{K} 2 \mathrm{OO}$ & 1.1 \\
\hline $\mathrm{Na}$ & $\%$ & 0.6 \\
\hline $\mathrm{Cu}$ & $\mathrm{ppm}$ & 42 \\
\hline $\mathrm{Fe}$ & $\mathrm{ppm}$ & 3500 \\
\hline $\mathrm{Zn}$ & $\mathrm{ppm}$ & 70 \\
\hline $\mathrm{Mn}$ & $\mathrm{ppm}$ & 230 \\
\hline
\end{tabular}

-- Valor no especificado

c. Compost producido por la empresa Alborada S. A. (CAL): el compost es producido para satisfacer el requerimiento de una finca independiente, el fundo Alborada, de 7 ha de cacao en producción y manejo del suelo con base en compost. No presenta una ficha técnica, sin embargo, mantiene un registro de los insumos usados en el compostaje: estiércol de cuy, estiércol de murciélago, restos de mazorcas de cacao, residuos vegetales frescos (tallo de plátano, hojas de kudzu y/o eritrina), mucilago de cacao y leche fresca.

d. Compost de residuos sólidos orgánicos: producidos por la municipalidad provincial de Leoncio Prado (CML). Este compost se produce con los residuos que la municipalidad recolecta a través del programa de segregación en la fuente, por medio de un recolector especialmente destinado a este fin. Los residuos son tratados mediante el compostaje en pilas aeróbicas y con aplicación de microorganismos eficientes, para mejorar el proceso y la calidad del producto.

\section{Caracterización fisicoquímica}

De cada compost identificado se extrajeron 4 muestras al azar y se realizó el análisis fisicoquímico correspondiente en el laboratorio de suelos de la Universidad Nacional Agraria de la Selva. Se determinó el \% de humedad por método gravimétrico, el \% de cenizas por calcinación, pH por método electrométrico, MO Walkley y Black, N micro Kjeldahl, P Olsen modificado, Ca, Mg, K y Na por acetato de amonio, y lectura en espectro fotómetro de absorción atómica; todos los análisis se realizaron siguiendo los protocolos descritos por Bazán (2017). 


\section{Análisis estadístico}

Es una investigación no experimental correlacional con ajuste estadístico completamente aleatorizado; los tratamientos lo constituyen los siguientes compost identificados: CAL, CMF, CML, CCD y CCF, y con tamaño de muestra $n=4$ (muestras por tipo de abono). Los datos se sometieron al análisis de varianza y la prueba post-hoc de Duncan con un nivel de significancia del 5 \% para comparar las medias de indicadores físicos y químicos entre los abonos. Para el procesamiento de los datos, se utilizó el software libre IBM-SPSS 25.

\section{Resultados}

\section{Caracterización físico-química}

Se evaluaron los principales indicadores fisicoquímicos (Tabla 3) que son considerados y exigidos por la NTC, la NOCh y la OMS. Además, son indicadores que definen la calidad de los abonos orgánicos tipo compost, señalados por diferentes autores.

Tabla 3. Estadística de indicadores fisicoquímicos en materia seca

\begin{tabular}{|c|c|c|c|c|c|c|c|}
\hline Indicador & CAL & CML & CCD & CMF & CCF & F & Sig \\
\hline Humedad & $34.8 \pm 17.2 \mathrm{a}$ & $32.28 \pm 19.24 \mathrm{a}$ & $33.55 \pm 16.9 \mathrm{a}$ & $28.2 \pm 16.56 \mathrm{a}$ & $27.28 \pm 17.5 \mathrm{a}$ & 306.39 & 0.96 \\
\hline Ceniza & $66.5 \pm 10.8 \mathrm{a}$ & $63.17 \pm 8.84 \mathrm{a}$ & $63.55 \pm 10.6 \mathrm{a}$ & $67.4 \pm 12.2 \mathrm{a}$ & $68.15 \pm 12.8 \mathrm{a}$ & 123.44 & 0.95 \\
\hline $\mathrm{pH}$ & $8.1 \pm 1.1 \mathrm{a}$ & $8.13 \pm 1.27 \mathrm{a}$ & $7.68 \pm 0.97 \mathrm{a}$ & $8.33 \pm 0.76 \mathrm{a}$ & $7.9 \pm 1.3 \mathrm{a}$ & 0.21 & 0.93 \\
\hline $\mathrm{MO}, \%$ & $33.5 \pm 10.8 \mathrm{a}$ & $36.84 \pm 8.84 \mathrm{a}$ & $36.45 \pm 10.6 \mathrm{a}$ & $32.62 \pm 12.2 \mathrm{a}$ & $29.35 \pm 9.3 \mathrm{a}$ & 107.99 & 0.84 \\
\hline $\mathrm{N}$ & $1.65 \pm 0.2 \mathrm{a}$ & $1.43 \pm 0.16 \mathrm{a}$ & $1.44 \pm 0.16 \mathrm{a}$ & $1.64 \pm 0.08 \mathrm{a}$ & $2.48 \pm 0.35 \mathrm{~b}$ & 18.03 & $<0.001$ \\
\hline $\mathrm{P} 205, \%$ & $1.58 \pm 0.5 \mathrm{a}$ & $1.57 \pm 0.44 \mathrm{a}$ & $1.35 \pm 0.82 \mathrm{a}$ & $1.2 \pm 0.28 \mathrm{a}$ & $1.76 \pm 0.5 \mathrm{a}$ & 0.29 & 0.64 \\
\hline $\mathrm{Ca} \%$ & $1.18 \pm 0.4 \mathrm{a}$ & $2.21 \pm 0.76 \mathrm{a}$ & $1.98 \pm 1.05 \mathrm{a}$ & $2.53 \pm 0.71 \mathrm{a}$ & $2.67 \pm 0.9 \mathrm{a}$ & 0.64 & 0.126 \\
\hline $\mathrm{Mg}, \%$ & $1.13 \pm 0.8 \mathrm{a}$ & $1.17 \pm 0.36 \mathrm{a}$ & $1.49 \pm 0.37 \mathrm{a}$ & $1.48 \pm 0.47 \mathrm{a}$ & $1.36 \pm 0.38 \mathrm{a}$ & 0.19 & 0.67 \\
\hline $\mathrm{K}, \%$ & $4 \pm 2 \mathrm{a}$ & $4.47 \pm 2.88 \mathrm{a}$ & $4.45 \pm 2.82 \mathrm{a}$ & $3.01 \pm 0.89 \mathrm{a}$ & $3.55 \pm 1.89 \mathrm{a}$ & 0.32 & 0.86 \\
\hline $\mathrm{Na} \%$ & $0.56 \pm 0.5 \mathrm{a}$ & $0.65 \pm 0.49 \mathrm{a}$ & $1.11 \pm 0.53 \mathrm{a}$ & $0.93 \pm 0.84 \mathrm{a}$ & $1.04 \pm 0.5 \mathrm{a}$ & 0.34 & 0.611 \\
\hline $\mathrm{Cu}, \mathrm{ppm}$ & $46.3 \pm 32.6 \mathrm{a}$ & $38.5 \pm 31.42 \mathrm{a}$ & $57.75 \pm 45.67 \mathrm{a}$ & $41 \pm 37.9 \mathrm{a}$ & $48.8 \pm 36.99 \mathrm{a}$ & 1387 & 0.954 \\
\hline $\mathrm{Fe}, \mathrm{ppm}$ & $5218 \pm 1071$ & $4421 \pm 2011$ & $2992 \pm 2162 \mathrm{a}$ & $4233 \pm 2765 \mathrm{a}$ & $5138 \pm 1206 \mathrm{a}$ & 379408 & 0.515 \\
\hline $\mathrm{Zn}, \mathrm{ppm}$ & $802 \pm 464$ & $727 \pm 440$ & $845 \pm 311 \mathrm{a}$ & $621.5 \pm 276 \mathrm{a}$ & $765 \pm 385 \mathrm{a}$ & 146398 & 0.935 \\
\hline $\mathrm{Mn}, \mathrm{ppm}$ & $576 \pm 329$ & $474 \pm 272$ & $648 \pm 286 \mathrm{a}$ & $399.5 \pm 211 \mathrm{a}$ & $507 \pm 323 \mathrm{a}$ & 82737 & 0.779 \\
\hline
\end{tabular}

Nota: CAL compost alborada, CML compost de la municipalidad de Leoncio Prado, CCD compost Cooperativa Divisoria, CMF compost de M\&F orgánicos, CCF compost comercial formulado, F valor de distribución, Sig significativo, valores promedios en la misma columna seguidos de letras diferentes indican diferencias significativas $(p<0,05)$ entre los tratamientos.

\section{Calidad de los compost evaluados}

\section{Según la nORMA tÉCNICA COLOMBIANA (NTC)}

La Tabla 4 muestra los niveles de indicadores fisicoquímicos encontrados y el control respectivo con la NTC, en ella se aprecia que el \% de cenizas en los diferentes casos es el único indicador que supera el nivel máximo fijado por la NTC (máximo 60 \%). Este indicador no tiene influencia sobre las funciones del compost como sustrato o enmienda, y no compromete la calidad del 
producto; por lo que, según esta norma, todos los compost evaluados presentan buena calidad, porque poseen valores adecuados de $\mathrm{pH}, \mathrm{MO}, \mathrm{N}, \mathrm{P}, \mathrm{K}$, Ca y Mg.

Tabla 4. Calidad del compost según la NTC 5167/2011

\begin{tabular}{|c|c|c|c|c|c|c|c|c|c|c|c|}
\hline \multicolumn{12}{|c|}{ Calidad según NTC } \\
\hline Indicador & NTC & CAL & Nivel & CML & Nivel & CCD & Nivel & CMF & Nivel & CCF & Nivel \\
\hline Humedad & $20-35 \%$ & 34.78 & * & 32.28 & * & 33.55 & * & 28.19 & * & 27.28 & * \\
\hline Cenizas & Max. $60 \%$ & 66.49 & ** & 63.17 & ** & 63.55 & $\star \star \star$ & 67.38 & ** & 68.15 & ** \\
\hline $\mathrm{pH}$ & $4-9$ & 8.1 & * & 8.13 & * & 7.68 & * & 8.33 & * & 7.9 & * \\
\hline MO & Min. $15 \%$ & 33.52 & * & 36.84 & * & 36.45 & * & 32.62 & * & 29.35 & * \\
\hline $\mathrm{N}$ & $>1 \%$ & 1.65 & * & 1.43 & * & 1.44 & * & 1.64 & * & 2.48 & * \\
\hline P205 & $>1 \%$ & 1.58 & * & 1.57 & * & 1.35 & * & 1.2 & * & 1.76 & * \\
\hline $\mathrm{Ca}$ & $>1 \%$ & 1.18 & * & 2.21 & * & 1.98 & * & 2.53 & * & 2.67 & * \\
\hline $\mathrm{Mg}$ & $>1 \%$ & 1.13 & * & 1.17 & * & 1.49 & * & 1.48 & * & 1.36 & * \\
\hline K & $>1 \%$ & 4 & * & 4.47 & * & 4.45 & * & 3.01 & * & 3.55 & * \\
\hline $\mathrm{Na}$ & -- & 0.56 & -- & 0.65 & -- & 1.11 & -- & 0.93 & -- & 1.04 & -- \\
\hline $\mathrm{Cu}$ & -- & 46.25 & -- & 38.5 & -- & 57.75 & -- & 41 & -- & 48.75 & -- \\
\hline $\mathrm{Fe}$ & -- & 5217.5 & -- & 4420.75 & -- & 2991.5 & -- & 4233 & -- & 5137.5 & -- \\
\hline $\mathrm{Zn}$ & -- & 801.5 & -- & 726.5 & -- & 845 & -- & 621.5 & -- & 764.5 & -- \\
\hline $\mathrm{Mn}$ & -- & 575.5 & -- & 474.25 & -- & 648 & -- & 399.5 & -- & 506.75 & -- \\
\hline \multicolumn{2}{|c|}{ Calidad del compost } & \multicolumn{2}{|c|}{ Buena $\left({ }^{\star}\right)$} & \multicolumn{2}{|c|}{ Buena $\left({ }^{\star}\right)$} & \multicolumn{2}{|c|}{ Buena $\left({ }^{*}\right)$} & \multicolumn{2}{|c|}{ Buena $\left(^{*}\right)$} & \multicolumn{2}{|c|}{ Buena $\left(^{*}\right)$} \\
\hline
\end{tabular}

Nota: CAL compost alborada, CML compost de la municipalidad de Leoncio Prado, CCD compost Cooperativa Divisoria, CMF compost de M\&F orgánicos, CCF compost comercial formulado, -- valor no especificado, * valor dentro del límite fijado por NTC, ** valor que excede al limite fijado por NTC.

La Tabla 5 muestra los niveles de indicadores físico-químicos encontrados y el control respectivo con la NOCh 2880, en ella se aprecia que los \% de humedad, pH, MO y Zn cumplen con los niveles exigidos para un compost de clase $\mathrm{B}, \mathrm{y}$ los indicadores $\mathrm{P}, \mathrm{K}, \mathrm{Ca}, \mathrm{Mg} \mathrm{Na}$ y Cu, los cumplen para un compost de clase A. Sin embargo, considerando que el \% de humedad compromete el peso del producto, y el pH y la MO son indicadores fundamentales que definen la reactividad y una serie de procesos químicos ligados a los grupos funcionales de la MO, definiendo en gran medida la calidad del compost, por tanto se clasifican en la clase B según la NOCh 2880.

Tabla 5. Calidad del compost según la NOCh 2880

\begin{tabular}{|c|c|c|c|c|c|c|c|c|c|c|c|c|}
\hline \multirow{2}{*}{ Indicador } & \multicolumn{12}{|c|}{ NOCh } \\
\hline & Clase A & Clase B & CAL & Clase & CML & Clase & CCD & Clase & CMF & Clase & $\mathrm{CCF}$ & Clase \\
\hline Humedad & $<25$ & $25-40 \%$ & 34.78 & B & 32.28 & B & 33.55 & B & 28.19 & B & 27.3 & B \\
\hline Ceniza & & & 66.49 & -- & 63.17 & -- & 63.55 & -- & 67.38 & -- & 68.2 & -- \\
\hline $\mathrm{pH}$ & $5-7.5$ & $<5 y>7.5$ & 8.1 & B & 8.13 & B & 7.68 & B & 8.33 & B & 7.9 & B \\
\hline MO & $>45 \%$ & $25-45 \%$ & 33.52 & B & 36.84 & B & 36.45 & B & 32.62 & $B$ & 29.4 & B \\
\hline $\mathrm{N}$ & $>0.8 \%$ & & 1.65 & A & 1.43 & A & 1.44 & A & 1.64 & A & 2.48 & A \\
\hline
\end{tabular}




\begin{tabular}{|c|c|c|c|c|c|c|c|c|c|c|c|}
\hline P205 & $<5 \%$ & 1.58 & A & 1.57 & A & 1.35 & A & 1.2 & A & 1.76 & A \\
\hline $\mathrm{Ca}$ & $>1 \%$ & 1.18 & A & 2.21 & A & 1.98 & A & 2.53 & A & 2.67 & A \\
\hline $\mathrm{Mg}$ & $>1 \%$ & 1.13 & A & 1.17 & A & 1.49 & A & 1.48 & A & 1.36 & A \\
\hline K & $>1 \%$ & 4 & A & 4.47 & A & 4.45 & A & 3.01 & A & 3.55 & A \\
\hline $\mathrm{Na}$ & $<1 \%$ & 0.56 & A & 0.65 & A & 1.11 & $\mathrm{~B}$ & 0.93 & A & 1.04 & B \\
\hline $\mathrm{Cu}$ & Max. 100 & 46 & A & 39 & A & 58 & A & 41 & A & 49 & A \\
\hline $\mathrm{Fe}$ & & 5218 & -- & 4421 & -- & 2992 & -- & 4233 & -- & 5138 & -- \\
\hline Zn & Max. 200 & 802 & $B$ & 727 & $\mathrm{~B}$ & 845 & $B$ & 622 & $B$ & 765 & B \\
\hline $\mathrm{Mn}$ & -- & 576 & -- & 474 & -- & 648 & -- & 400 & -- & 507 & - \\
\hline \multicolumn{2}{|c|}{ Calidad del compo } & \multicolumn{2}{|c|}{ Clase B } & \multicolumn{2}{|c|}{ Clase B } & \multicolumn{2}{|c|}{ Clase B } & \multicolumn{2}{|c|}{ Clase B } & \multicolumn{2}{|c|}{ Clase B } \\
\hline
\end{tabular}

Nota: CAL compost alborada, CML compost de la municipalidad de Leoncio Prado, CCD compost Cooperativa Divisoria, CMF compost de M\&F orgánicos, CCF compost comercial formulado, -- valor no especificado, A y B compost de alta calidad y calidad media según la NOCh.

La Tabla 6 muestra que los diferentes compost evaluados presentan medias que, según la $\mathrm{O}$ MS, son de buena calidad.

Tabla 6. Calidad del compost según OMS

\begin{tabular}{|c|c|c|c|c|c|c|c|c|c|c|c|}
\hline \multicolumn{12}{|c|}{ Calidad según NTC } \\
\hline Indicador & OMS & CAL & Nivel & CML & Nivel & CCD & Nivel & CMF & Nivel & CCF & Nivel \\
\hline Humedad & $30 \%-50 \%$ & 34.78 & * & 32.28 & * & 33.55 & * & 28.19 & $\star$ & 27.28 & * \\
\hline $\mathrm{pH}$ & $6-9$ & 8.1 & * & 8.13 & * & 7.68 & * & 8.33 & * & 7.9 & * \\
\hline MO & $25-50 \%$ & 33.52 & * & 36.84 & * & 36.45 & * & 32.62 & * & 29.35 & * \\
\hline $\mathrm{N}$ & $0.4 \%-3.5 \%$ & 1.65 & * & 1.43 & * & 1.44 & * & 1.64 & * & 2.48 & * \\
\hline $\mathrm{P}_{2} \mathrm{O}_{5}$ & $0.3 \%-1.8 \%$ & 1.58 & * & 1.57 & * & 1.35 & * & 1.2 & * & 1.76 & * \\
\hline $\mathrm{Ca}$ & -- & 1.18 & -- & 2.21 & -- & 1.98 & -- & 2.53 & -- & 2.67 & -- \\
\hline $\mathrm{Mg}$ & -- & 1.13 & -- & 1.17 & -- & 1.49 & -- & 1.48 & -- & 1.36 & -- \\
\hline $\mathrm{K}$ & -- & 4 & -- & 4.47 & -- & 4.45 & -- & 3.01 & -- & 3.55 & -- \\
\hline $\mathrm{Na}$ & -- & 0.56 & -- & 0.65 & -- & 1.11 & -- & 0.93 & -- & 1.04 & -- \\
\hline \multicolumn{2}{|c|}{ Calidad del compost } & \multicolumn{2}{|c|}{ Buena $\left({ }^{*}\right)$} & \multicolumn{2}{|c|}{ Buena $\left(^{*}\right)$} & \multicolumn{2}{|c|}{ Buena $\left(^{\star}\right)$} & \multicolumn{2}{|c|}{ Buena $\left({ }^{*}\right)$} & \multicolumn{2}{|c|}{ Buena $\left({ }^{\star}\right)$} \\
\hline
\end{tabular}

Nota: CAL compost alborada, CML compost de la municipalidad de Leoncio Prado, CCD compost Cooperativa Divisoria, CMF compost de M\&F Orgánicos, CCF compost comercial formulado, -- valor no especificado, * nivel dentro de los límites fijados por la OMS.

\section{Discusión}

\section{Indicadores fisicoquímicos}

Los resultados (Tabla 3) muestran valores de humedad entre 27.28 (CCF) y $34.8 \%$ (CAL). Este parámetro es importante porque un exceso de humedad afectaría el cálculo y la cantidad aplicada al suelo; por ello, la FAO (2013) y la NOCh recomiendan que no debe superar el $40 \%$; la NTC recomienda un rango de 20 a $35 \%$, y la OMS, hasta $50 \%$. Los valores encontrados están dentro de estos rangos y no se observan diferencias estadísticas entre los compost evaluados.

El pH (Tabla 3) varía de 7.68 (CCD) a 8.33 (CMF); este indicador es fundamental para determinar la calidad de un compost, la cual está influenciada por la composición de los resi- 
duos utilizados en el proceso de compostaje (Florida \& Reategui, 2019; FAO, 2013) que tiende a incrementarse con el tiempo de compostaje (Bárbaro et al., 2019), y se recomiendan valores que fluctúen en la neutralidad (Rivas \& Silva, 2020). Los resultados coinciden con Rivas \& Silva (2020), quienes encontraron valores entre 6.6 a 7.3 con residuos de jardinería, pergamino de café y bora; también Florida \& Reategui (2019), en compost de plumas y estiércol de vacuno registra medias de 7.79 a 8.32; Bárbaro et al. (2019) reportan valores entre 4.7 a 8.7 en 15 compost de diferentes orígenes; Hernández et al. (2013), utilizando mezclas de estiércol de ganado vacuno lechero, estiércol de gallina, aserrín y esquilmos de maíz, encontraron medias entre 7.8 a 8.1 de $\mathrm{pH}$; Widman et al. (2005) reportan una media de $7.35 \mathrm{de} \mathrm{pH}$ en compost producido a partir de residuos sólidos urbanos; Olivares et al. (2012) señalan medias de 6 a 7.42 de pH en lombricomposta y compost de estiércol de ganado vacuno lechero. Además, están dentro de los rangos establecidos por la NTC (4-9), la NOCh (pH >7.5), la OMS (6-9) y la FAO (2013) que fija un rango de 6.5 a 8.5 de pH y no presentan diferencias estadísticas entre los compost evaluados.

La MO presentó medias de 29.35 (CCF) a 36.84 \% (CML). No se encontraron diferencias estadísticas para este indicador (Tabla 3 ) y el resultado cumple con los rangos establecidos por la NTC (mínimo $15 \%$ ), la NOCh (25-45 \%), la OMS (25 a50 \%) y la FAO (2013) que exige valores mayores a $20 \%$. Además, coincide con los resultados de Olivares (2012), quien reporta medias de 25.7 a $32.01 \%$; Widman et al. (2005) reportan una media de 38.46 \%; Hernández et al. (2013) de 18.79 a 30.68 \% de M0; Vázquez \& Loli (2018) reportan una media para vermicompost y compost de 37.75 y 40.73 respectivamente; Mendoza (2012) medias entre 32 y 38 \% de MO en compost de residuos vegetales. Sin embargo, son inferiores a lo reportado por Rafael (2015) de 39 a 59.8 \%; Florida \& Reategui (2019) 39.6 a 51.14 \% de MO, en combinación de estiércol de vacuno y plumas de pollo, y Florida et al. (2016) medias entre 61.3 a 92. 27 \% de M0, en compost con diferentes insumos, cáscara de plátano, pulpa de café y escobajo de palma.

El N presenta medias entre 1.43 (CML) a 2.48 \% (CCF), encontrándose diferencias estadísticas entre los compost evaluados (Tabla 3) y son valores normales, excepto el CCF que presenta un valor superior al resto con $2.48 \%$; este último es superior a lo reportado por Mendoza (2012), con medias entre 1.05 y $1.33 \%$ de $\mathrm{N}$ en compost de residuos vegetales, y por Soriano (2016), quien reporta medias entre 1.27 a 1.54 \% N. Además, los resultados son inferiores a lo encontrado por Rafael (2015), medias de 1.79 a $3.02 \%$ de N, y de Florida \& Reategui (2019), medias de 2.4 a $4.8 \%$ de N. Respecto a los $2.48 \%$ de $\mathrm{N}$ en el compost CCF, es un valor alto para este compost; teniendo en cuenta que es el que presentó menor \% de MO (29.35\%), se presume una posible alteración con alguna fuente química nitrogenada. Sin embargo, los valores se encuentran dentro de los rangos establecidos por la OMS $0.4-3.5 \%$; la NOCh, > 0.8 \%, y la NTC que exige mayor a $1 \%$ de $\mathrm{N}$.

Los macroelementos P205 (1.2 a 1.76 \%), Ca (1.18 a $2.67 \%)$, Mg (1.13 a 1.49 \%), K (4 a $4.47 \%)$ y $\mathrm{Na}$, con medias entre 0.56 a $1.11 \%$, no muestran diferencias estadísticas en estos indicadores entre los diferentes compost evaluados (Tabla 3). Los resultados son similares a los reportados por Alvarez et al., 2019, Florida \& Reategui (2019), Vázquez \& Loli (2018), López et al. (2017), Soriano (2016), Florida et al., (2016); Rafael (2015), Hernández et al. (2013) y Olivares (2012), y están dentro de los parámetros exigidos por la NTC y la NOCh, que exigen para estos indicadores valores mayores a $1 \%$.

Los metales pesados no mostraron diferencias significativas y se encontraron medias de 38.5 (CML) a 57.75 ppm de Cu (CCD), 2992 (CCD) a 5218 ppm de Fe (CAL), 621.5 (CMF) a 802 ppm de Zn (CAL) y 399.5 (CMF) a 648 ppm de Mn en el compost CCD. Los resultados son similares a 
lo reportado por Rafael (2015) en todos los indicadores; en el caso de Fe y Cu son similares a lo encontrado por Florida \& Reategui (2019) medias entre 3738 a 5290 ppm de Fe y 42 a 49 ppm de Cu, excepto Zn y Mn son medias más bajas; también Hernández et.al., (2013) muestran valores similares en Cu (48 a 93 ppm), pero menores en Zn, Mn y Fe que nuestros resultados. Sin embargo, Alvarez et al. (2019) y Olivares (2012) encontraron medias menores en todos los metales evaluados. De acuerdo con la NTC, no se puede hacer el control de calidad, ya que no precisa los rangos límites y la NOCh exige rangos de Cu (max 100) y Zn (max 200); en este caso se cumple con los niveles de Cu, mas no con los niveles de Zn. Sin embargo, los valores encontrados, según los autores citados, son medias aceptables que no comprometen negativamente la calidad del compost.

\section{Calidad del compost según las normas}

Se evaluó como indicador físico la humedad y químicos tales como cenizas, pH, MO, los macronutrientes $\mathrm{P}_{2} \mathrm{O}_{5}, \mathrm{~K}$, Ca, Mg y Na y los microelementos o metales pesados $\mathrm{Cu}$, Fe, Mn y Zn. Estos son indicadores que nos permiten determinar la calidad y han sido utilizados por diferentes autores (Hernández, 2020; Rivas \& Silva, 2020; Florida \& Reategui, 2019; Muscolo et al., 2018; Cabrera \& Rossi, 2016; Florida et al., 2016; Rafael, 2015; García et al. 2014; FAO, 2013; Mendoza, 2012; Olivares, 2012).

Los resultados (Tabla 4 y 6 ) muestran que todos los indicadores evaluados cumplen con los rangos establecidos por la NTC y la OMS, excepto el \% de cenizas para la NTC; al respecto, los valores altos en cenizas manifiestan la presencia de material mineral en el producto, no obstante, ninguno de los autores citados considera que esto comprometa la calidad del compost, por lo que los resultados, según la NTC 5167 y la OMS, corresponden a compost de buena calidad. Sin embargo, para la NOCh 2880, los resultados (Tabla 5) muestran que el \% de humedad, el pH, MO y los niveles de Zn corresponden a compost de clase B. Considerando al pH y la MO como indicadores fundamentales que definen la reactividad y una serie de procesos químicos que definen en gran medida la calidad del compost (Florida \& Reategui, 2019; Cabrera \& Rossi, 2016; Rafael, 2015; García et al. 2014; FAO, 2013), para la NOCh 2880 todos los compost evaluados y que se comercializan en el distrito Rupa Rupa son de clase B, compost de calidad media, y que presenta ciertas restricciones para su uso.

\section{Conclusión}

Se identificó a cinco productos tipo compost que son comercializados en el distrito. Los indicadores fisicoquímicos no presentan diferencias estadísticas entre los compost evaluados, excepto para los niveles de Nitrógeno. Los valores medios de los diferentes indicadores se encuentran dentro de los rangos establecidos por la NTC, la NOCh, la OMS y la FAO, y son valores que coinciden con las referencias citadas.

Los resultados, según la NTC 5167 y la OMS, corresponden a compost de buena calidad y, según la NOCh 2880, se clasifica a todos los compost evaluados como compost de calidad media y que presenta restricciones para su uso.

La norma con mayor exigencia y jerarquización, en los diferentes indicadores fisicoquímicos, que mejor determina la calidad del compost es la NOCh 2880, que puede ser aplicada en países que aún no disponen de una norma específica. 


\section{Referencias}

Alvarez, V. M. Largo, A., Iglesias, A. S., \& Castillo J. (2019). Calidad de compost obtenido a partir de estiércol de gallina, con aplicación de microorganismos benéficos. Scientia Agropecuaria, 10(3), 353361. http://dx.doi.org/10.17268/sci.agropecu.2019.03.05

Bárbaro, L., Karlanian, M., Rizzo, P., \& Riera, N. (2019). Caracterización de diferentes compost para su uso como componente de sustratos. Chilean Journal of Agricultural \& Animal Sciences, 35(2), 126136. https://dx.doi.org/10.4067/S0719-38902019005000309

Bazán, R. (2017). Manual de procedimientos de los análisis de suelos y agua con fines de riego. Ministerio de Agricultura y Riego, Instituto Nacional de Innovación Agraria. Instituto Nacional de Innovación Agraria, pp. 92 https://bit.ly/34UirxL

Cabrera, V., \& Rossi, M. (2016). Propuesta para la elaboración de compost a partir de los residuos vegetales provenientes del mantenimiento de las áreas verdes públicas del distrito de Miraflores. [Tesis de pregrado, Universidad Nacional Agraria la Molina]. Repositorio UNALM. https://bit. ly/30Zt3KC

Florida, N., \& Reategui, F. (2019). Compost a base de plumas de pollos (Gallus domesticus). Livestock Research for Rural Development, 31(11), s. p. https://bit.ly/3nJQt08

Florida, N., Reategui, F., \& Pocomucha, V. (2016). Caracterización del compost a base de plumas de pollos (Gallus domesticus) y otros insumos. Investigación y Amazonía. 6(2), 1-5. https://bit.ly/340iQS5

García, D., Lima, L., Ruíz, L., \& Calderón, P. (2014). Métodos y parámetros para determinar la madurez en el compost a nivel de Fincas. Medio Ambiente y Desarrollo, 14(26), s. p. https://bit.ly/3nHElg4

Hernández, M. (2020). Aspectos físico-químicos, bioquímicos y microbiológicos del proceso de compostaje. Evaluación de la calidad. Instituto de Recursos Naturales y Agrobiología de Salamanca. https://bit.ly/36YjrU3

Hernández, R. O., Hernández, T. A., Rivera, F. C., Arras, V. A., \& Ojeda B. D. (2013). Calidad nutrimental de cuatro abonos orgánicos producidos a partir de residuos vegetales y pecuarios. Terra Latinoamericana, 37(1), 35-46. https://bit.ly/3nLDDP5

López, B., Andrade, R., Herrera, S. M., González, C. O., \& García de la F. C. (2017). Propiedades de un compost obtenido a partir de residuos de la producción de azúcar de caña. Revista Centro Agrícola, 44(3), 49-55. https://bit.ly/33PzFg8

Mendoza, M. (2012). Propuesta de compostaje de los residuos vegetales generados en la Universidad de Piura. [Tesis de pregrado, Universidad de Piura]. Repositorio PIRHUA. https://handle. net/11042/1728

Ministerio de Agricultura y Riego. (2019a). Situación actual del café en el país. https://bit.ly/2Flz24y

Ministerio de Agricultura y Riego, (Enero 2019b). Observatorio de Commodities: Cacao. Boletín de publicación trimestral. Dirección General de Políticas Agrarias, Dirección de Estudios Económicos e Información Agraria. https://bit.ly/36UWbGx

Ministerio de Agricultura y Riego. (2019c). Comportamiento del mercado nacional e internacional de los commodities. Dirección General de Políticas Agrarias, Dirección de Estudios Económicos e Información Agraria. https://bit.ly/34ROqP2

Ministerio de Agricultura y Riego. (2016). Estudio del cacao en el Perú y en el mundo: Situación actual y perspectivas en el mercado nacional e internacional al 2015. MINAGRI-DGPA-DEEIA. https://bit. ly/34Lgqnm

Muscolo A., Papalia T., Settineri G., Mallamaci C., \& Kaczanowska A. (2018). Are Raw Materials or Composting Conditions and Time That Most Influence the Maturity and/or Quality of Composts? Comparison of Obtained Composts on Soil Properties. Journal of Cleaner Production, 195, 93-101. https://doi.org/10.1016/j.jclepro.2018.05.204

Instituto Nacional de Normalización. (2004). Norma Chilena de Calidad de Compost (Norma Chilena Oficial 2880). https://bit.ly/34PjmPU 
Instituto Colombiano de Normas Técnicas y Certificación. (2011). Productos para la industria agrícola: Productos orgánicos usados como abonos o fertilizantes y enmiendas o acondicionadores de suelo (Norma Técnica Colombiana 5167). Segunda actualización . p.10. https://bit.ly/36YoML5

Olivares, C., Hernández, R. A., Vences, C. C, Jáquez, B., \& Ojeda, B. D. (2012). Lombricomposta y composta de estiércol de ganado vacuno lechero como fertilizantes y mejoradores de suelo. Trópico Húmedo, 28(1): 27-37. https://bit.ly/30ZX9xi

Organización de las Naciones Unidas para la Alimentación y la Agricultura (2013). Manual de compostaje del agricultor. Experiencias en América Latina Oficina Regional para América Latina y el Caribe Santiago de Chile. https://bit.ly/3dijhro

Peralta, A. N., Freitas, G. B., Watthier, M., \& Silva, S. R. (2019). Compost, bokashi y microorganismos eficientes: Sus beneficios en cultivos sucesivos de brócolis. Idesia (Arica), 37(2), 59-66. https://dx.doi. org/10.4067/S0718-34292019000200059

Pulgar, V. (2014). Las ocho regiones naturales del Perú. Terra Brasilis. Revista da Rede Brasileira de História da Geografia e Geografia Histórica, 3 [en línea], s. p. https://doi.org/10.4000/terrabrasilis.1027

Rafael, A. (2015). Proceso de producción y aplicación del producto microorganismos eficaces en la calidad de compost a partir de la mezcla de tres tipos de residuos orgánicos, Sapallanga-Huancayo. [Tesis de pregrado, Universidad Nacional del Centro del Perú]. Repositorio UNCP. https:// bit.ly/3nJ5hMx

Ramos, A., \& Terry, E. (2014). Generalidades de los abonos orgánicos: importancia del bocashi como alternativa nutricional para suelos y plantas. Cultivos Tropicales, 35(04): 52-59. https://bit. ly/370uigm

Rivas, N., \& Silva, A. R. (2020). Calidad física y química de tres compost, elaborados con residuos de jardinería, pergamino de café y bora (Eichhornia crassipes). Ciencia Unemi, 13(32), 87-100. https:// bit.ly/372lvt1

Sharma A., Saha T. N., Arora A., Shah R., \& Nain L. (2017). Efficient Microorganism Compost Benefits Plant Growth and Improves Soil Health in Calendula and Marigold. Horticultural Plant Journal, 3(2), 67-72. http://dx.doi.org/10.1016/j.hpj.2017.07.003

Servicio Nacional de Meteorología e Hidrología del Perú. (2020). Descarga de datos meteorológicos a nivel nacional. https://bit.ly/3IDD7Ax

Soriano, J. (2016). Tiempo y calidad del compost con aplicación de tres dosis de "microorganismos eficaces"- concepción. [Tesis de pregrado, Universidad Nacional del Centro del Perú]. Repositorio UNCP. https://bit.ly/30wOVaT

Vázquez, J., \& Loli, O. (2018). Compost y vermicompost como enmiendas en la recuperación de un suelo degradado por el manejo de Gypsophila paniculata. Scientia Agropecuaria, 9(1) 43-52. http:// doi/10.17268/sci.agropecu.2018.01.05

Widman, A., Herrera, R., \& Cabañas, V. D. (2005). El uso de composta proveniente de residuos sólidos municipales como mejorador de suelos para cultivos en Yucatán. Estudios preliminares. Ingeniería, 9(3): 31-38. https://bit.ly/310A8dB 